\title{
The Effect of Carica Papaya Leaf Extract on Increasing Platelet Count Among Dengue Fever Patients: A Meta-Analysis
}

\author{
Josephine Yuson-Sunga ${ }^{1, *},[M D]$ \\ ORCID: 0000-0002-0252-2422 \\ Faith Abigail Co,", , [MD] \\ ORCID: 0000-0002-6879-4105
}

R-jay F. Agbon Falalimpa Agbon', [MD]

ORCID: 0000-0001-7779-1494

Michelle Pia Anne Boco Austria', [MD]

ORCID: 0000-0002-6637-3075

Nadine Viloria Caballes', [MD]

ORCID: 0000-0002-2706-3584

Carl Earvin Lee Favorito', [MD]

ORCID: 0000-0002-5503-4764

Camille Cleo Leones Lamera', [MD]

ORCID: 0000-0003-0222-7623

Alyanna Justine Lustado Manuba', [MD]

ORCID: 0000-0001-9251-3150

Diego Louise David Ramos', [MD]

ORCID: 0000-0003-2245-9026

Melizza Joaquin Soriano', [MD]

ORCID: 0000-0001-5242-4321

Lilette de los Santos Torrena', [MD]

ORCID: 0000-0002-8644-3050

Claudine Calimlim Villostas', [MD]

ORCID: 0000-0001-7062-1679

'Department of Clinical Research, St. Luke's Medical Center College of Medicine-William H. Quasha Memorial

* These authors contributed equally to this work.

This paper has been presented during the 14th Congress of Asian Society for Pediatric Research "Emerging and Relevant Issues in Child Health" in October 2018, Manila, Philippines

Corresponding Author (1): Josephine Yuson-Sunga, St. Luke's Medical Center College of Medicine-William H. Quasha Memorial

E-mail:marijoysmd@gmail.com

Corresponding Author (2): Faith Abigail Co

St. Luke's Medical Center College of Medicine-William

H. Quasha Memorial

E-mail: faithabigailkco@gmail.com

https://doi.org/10.32552/2021.ActaMedica.457

\section{n ABSTRACT Con}

Objective: Hemorrhagic fever is a very serious complication of dengue, which remains a major public health concern in the Philippines. On average, there are 170, 503 symptomatic dengue infections and 750 deaths reported in 4 years, with an incidence of around 180 dengue episodes per 100,000 , and a case fatality rate of approximately $0.44 \%$, owing mostly to bleeding secondary to thrombocytopenia, with reported mortalities occurring among individuals less than 20 years of age. In most rural areas in the country, a health care institution is often inaccessible and most patients cannot afford the high cost of hospitalization and transfusion. In a local study of the knowledge, attitude, and practices on dengue, herbal medicines including "tawatawa" and papaya are often used as an alternative supportive treatment due to easy availability and low cost. Animal studies also support a potential therapeutic effect. The objective of this review is to assess the effects of $C$. papaya leaf extract as an adjunct treatment among patients with dengue hemorrhagic fever using a systematically searched and synthesized meta-analysis.

Materials and Methods: Six randomized clinical trials with a total of 988 subjects involving the efficacy of $C$. papaya leaf extract in increasing platelet count in dengue patients were searched through PubMed, Google Scholar, Scopus, and Science Direct and selected after assessing the eligibility and validity of the articles through a standard criterion. Mean difference of platelet counts from days 1 to 5 were pooled together and analyzed by Review Manager (RevMan) software 5.3.

Results: Pooled estimates revealed significant increase in platelet count at Day $3(\mathrm{MD}=12.18 ; \mathrm{Cl}$ 10.28-14.08), Day $4(\mathrm{MD}=31.30 ; \mathrm{Cl}$ 27.77-34.83), and Day 5 (MD=13.23; Cl 9.90-16.55). Random effects model at Day 5 also showed significant increase in platelet count. Likewise, a subgroup analyses of studies based on route of administration, frequency, and dosage were performed and showed significant increase in platelet count.

Conclusion: Based on this meta-analysis, supplementation with $C$. papaya leaf extract in patients with dengue shows a beneficial effect in increasing platelet count.

Keywords: Dengue fever, dengue, blood platelet, carica, carica papaya, thrombocytopenia 


\section{INTRODUCTION}

Dengue fever is an acute infectious viral disease transmitted via a bite of an infected female Aedes mosquito. It is usually seen in infants and young children but it can affect anyone at any age. It manifests as high fever, severe headache, myalgia and arthralgia, rash, nose bleeding, and gastrointestinal bleeding, which could eventually lead to massive bleeding, shock, and even death if not managed properly. In fact, dengue hemorrhagic fever, if left untreated, has a mortality rate of $10-20 \%$ with an even higher mortality rate if it progresses to dengue shock syndrome (DSS) [1].

Dengue infects about 2.5 billion people worldwide - 975 million of which belongs to tropical and subtropical countries in Southeast Asia, the Pacific, and the America [2].

The Philippines ranked seventh with the highest number of dengue fever cases in the world between 2004 and 2010. The Department of Health - Epidemiology Bureau reported a $41 \%$ increase in cases of dengue fever in the Philippines from January 1, 2016 to June 11, 2016 compared to the 2015 data [3].

In addition to its significant effect on morbidity and mortality, dengue also contributes to the economic burden of diseases in our country. According to a study by Edillo et al (2015), direct medical costs of clinically diagnosed dengue cases in the Philippines is estimated to be $\$ 345$ million (in 2012 US dollars) in a year and appears to have overtaken malaria in terms of economic impact [4].

Infection with dengue virus can either be primary or secondary. Primary infection results to an acute febrile illness known as dengue fever which is cleared in a week by a complex immune response. It causes fever of three to fivedays duration, usually accompanied by headache, rashes on the body with some thrombocytopenia, and relative leukopenia. Other symptoms may include severe joint and muscular pain (breakbone fever), nausea, vomiting, and retro-orbital eye pain. Secondary infection is more severe and results in hemorrhagic fever or dengue shock syndrome. This complication is characterized by increased vascular permeability and plasma leakage leading to hemorrhagic manifestations and severe thrombocytopenia, the exact mechanism of which is not clearly known [5].
In a local study of the knowledge, attitude, and practices on dengue, herbal medicines including "tawa-tawa" and papaya are often used as an alternative supportive treatment due to easy availability and low cost [6]. According to the folk medicine, papaya latex can cure dyspepsia and is also useful for external burns and scalds. Papaya seeds and fruits are excellent anthelminthic and anti-amoebic agents [7]. The latex, ripe fruits, unripe fruits, seeds, seeds juice, root, leaves, flower, and stem bark of $C$. papaya are used as antimicrobial, anthelminthic, antimalarial, antifungal, antiamoebic, hepatoprotective, for male and female antifertility, as an immunomodulatory and against allergies [8]. Animal studies show that papaya leaf extracts have a potential therapeutic effect on disease processes causing destabilization of biological membranes as they inhibit hemolysis in vitro [9]. However, only a few studies have been conducted in humans and the results are inconsistent.

Given the potential of this alternative approach, this review aims to assess the effects of Carica papaya leaf extract as an adjunct in the treatment of dengue fever patients. Specifically, this aims (1) to identify, retrieve, and assess all studies possible, evaluating the effects of $C$. papaya leaf extract in increasing the mean platelet count among dengue patients and (2) to assess the efficacy of the extract in increasing the mean platelet count over time (day 1 to day 5) among dengue patients.

\section{MATERIALS and METHODS}

\section{Search Methods for Identification of Studies}

Electronic searches of the literature were conducted in the following databases: PubMed, Google Scholar, Scopus, and ScienceDirect. The search terms used included "Carica papaya", "dengue", "platelet count", and derivatives of these. Only randomized, placebo-controlled studies involving human subjects of any age group were included in this paper. The retrieved articles were subjected to critical appraisal using the standard criteria for validity and applicability and the inclusion criteria set by the investigators. 


\section{Selection and Data Extraction}

The following items were recorded from the included trials: severity of dengue fever, initial platelet count prior to intervention (Carica papaya extract), daily platelet count during treatment, and dose, form, and duration of intervention.

Eligibility of studies were assessed by at least 2 independent reviewers. Data were extracted systematically by two independent reviewers according to the patient's characteristics, interventions, and results. Disagreements were resolved through group discussion. All identified trials were listed and trials excluded from the metaanalysis were identified with reason for exclusion.

\section{Methodological Quality}

The articles were appraised for validity using the following primary and secondary guidelines. The guides for the primary criteria were: (1) was the study randomized; (2) were all patients who entered the trial properly accounted for and attributed at its conclusion; (3) was follow up complete; and (4) were patients analyzed in the groups which they are randomized? On the other hand, the guides for the secondary criteria were: (1) were the subjects, investigators, or outcome blinded; (2) were groups similar at the start of the trial; and (3) were the groups treated equally? All six studies met both primary and secondary criteria, garnering ratings of $A$.

\section{Statistical Methods}

Studies were performed according to intention-totreat analysis, such that all patients were analyzed in the groups to which they were randomized. The dependent variable consisted of a continuous outcome, the mean platelet difference, taken over time, with their corresponding standard deviations. They were divided into the control and experimental groups, with the number of participants taken into account for each group per study. We then conducted analyses using random effects model initially and further did sub-analyses among those with heterogeneity.

The statistical package RevMan Analyses 5.3 was used. Since this study dealt with continuous data, the weighted mean difference (WMD) with a $95 \%$ confidence interval $(\mathrm{Cl})$ was used.

\section{RESULTS}

\section{Search Results}

The initial search yielded a total of 113 results from the four largest scientific databases: PubMed (5), Google Scholar (96), Scopus (3), ScienceDirect (9). Duplicate articles were identified and subtracted, leaving a remainder of 98 articles. Limits were applied to include only articles with titles and abstracts pertaining to randomized clinical trials on humans of any age group.

Nine full-text articles were obtained and further assessed for eligibility. Three studies were excluded because they did not meet the inclusion criteria. Six full-text articles in total were included in this metaanalysis.

\section{Included Trials}

Six randomized clinical trial full-text articles (Abhishek et al., 2015, Gadwhal et al., 2015; Gowda et al., 2014; Khan Assir et al., 2011; Subenthiran et al., 2013; Yunita et al., 2012) fulfilled the inclusion criteria.

The inclusion criteria of the randomized clinical trials varied in terms of age range and initial platelet counts but inclusion criteria were generally of good quality and all were confirmed cases of dengue fever or dengue hemorrhagic fever.

The interventions all included Carica papaya leaf extracts but dosage and preparations varied, with two studies using the same $1100 \mathrm{mg}$ tablet form, one using $500 \mathrm{mg}$ capsule, one using $550 \mathrm{mg}$

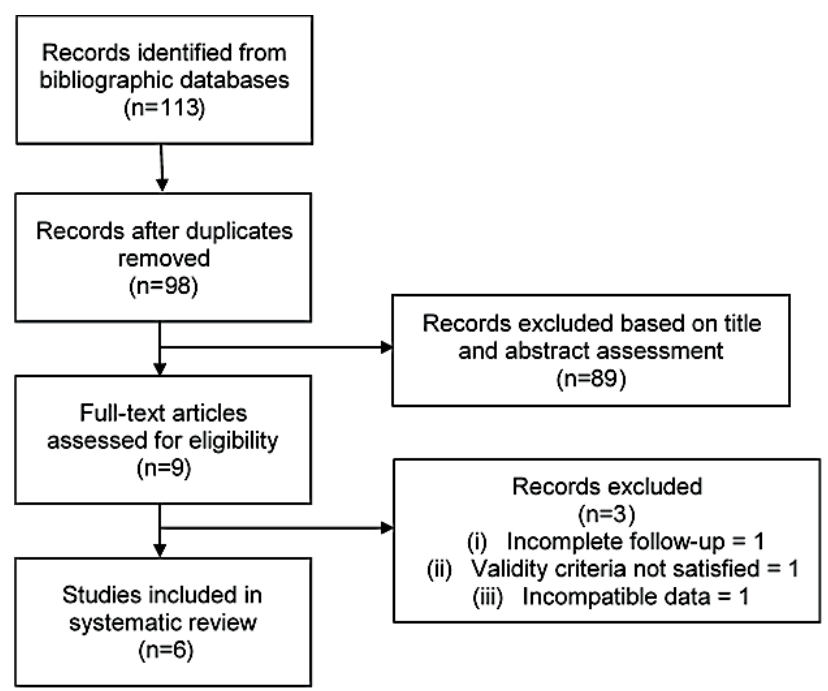

Figure 1. Flowchart of Search Methods for Identification of Studies 
capsule, and others prepared on-site as juice or syrup. Frequency and duration of intervention also varied among studies, with median duration of 5 days.

All patients concurrently underwent standard supportive treatment for dengue, with one study by Khan Assir et al. making use of placebo in addition to standard treatment.

\section{Excluded Studies}

A total of 89 non-duplicate studies were excluded from the initial search, many of which were case reports, in-vitro studies, or murine studies. After the application of limits, nine articles were retrieved. From these nine studies, three were excluded for the following reasons: (1) one study by Bajaj et al. had incomplete follow-up (2) one study by Hettige et al. did not satisfy the validity criteria, and (3) one study by Kasture et al. did not provide data compatible for analysis.

\section{Change in Platelet Count Measured at Days 1 and 2}

The efficacy of papaya in increasing platelet count was measured by comparing six randomized clinical trials with the control from days 1 to 5 . Among the six included studies, no study reported results favoring the $C$. papaya group.

However, pooled estimates from day 2 showed significant effect $(M D=1.96 ; C l=0.56,3.36)$ in increasing platelet count as compared to the control group. There was no significant heterogeneity detected.

\section{Change in Platelet Count Measured at Day 3}

Among the six studies included, two studies showed results that strongly favor the C. papaya group at day 3. The four other studies tend to favor the treatment group. The results were favorable to the treatment group that pooled estimates of the study showed significant effect $(M D=12.18$; $\mathrm{Cl}=10.28,14.08)$ in increasing the platelet count as compared to the control group. There was no significant heterogeneity detected $\left(I^{2}=49 \%\right)$.

\section{Change in Platelet Count Measured at Day 4}

At day 4 , the study by Subenthiran et al was not included because they did not report data beyond day 3. Among the included studies, three of them strongly favored the C. papaya group at day 4 . The results from the two other studies tend to favor the treatment group. Pooled estimates again favored the C. papaya group showing significant effect in increasing platelet count $(\mathrm{MD}=31.30 ; \mathrm{Cl}=27.77$, 34.83) as compared to the control group. The mean difference was highest at this day. There was no significant heterogeneity detected $\left(I^{2}=45 \%\right)$.

\section{Change in Platelet Count Measured at Day 5}

At day 5 of treatment, there was still significant increase in platelet count in the C. papaya group

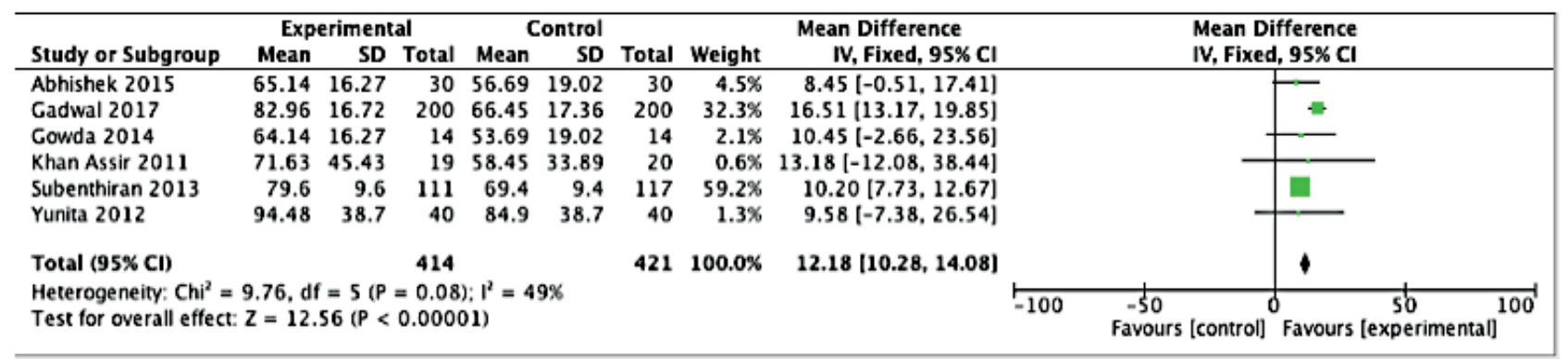

Figure 2. Efficacy of C. papaya in increasing the platelet count measured at Day 3.

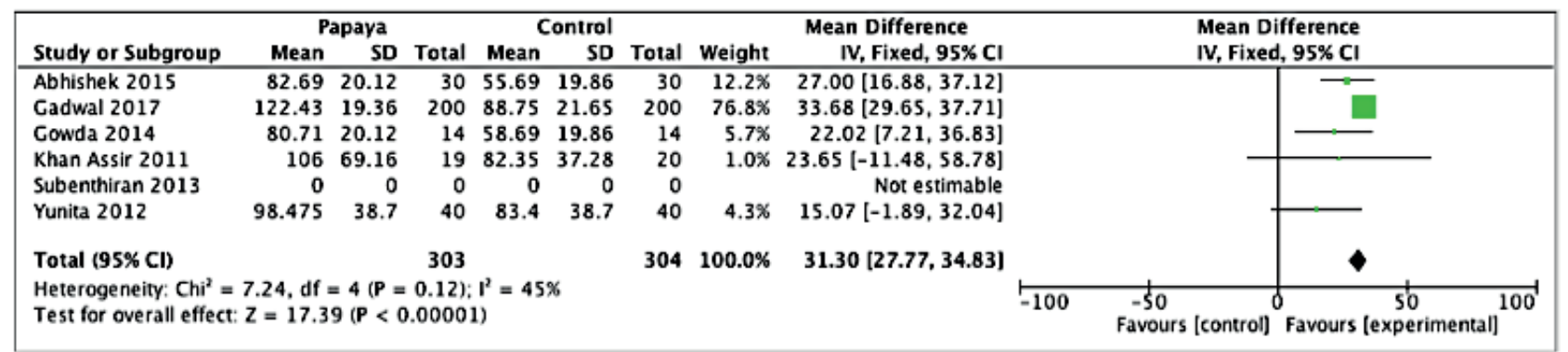

Figure 3. Efficacy of C. papaya in increasing the platelet count measured at Day 4. 


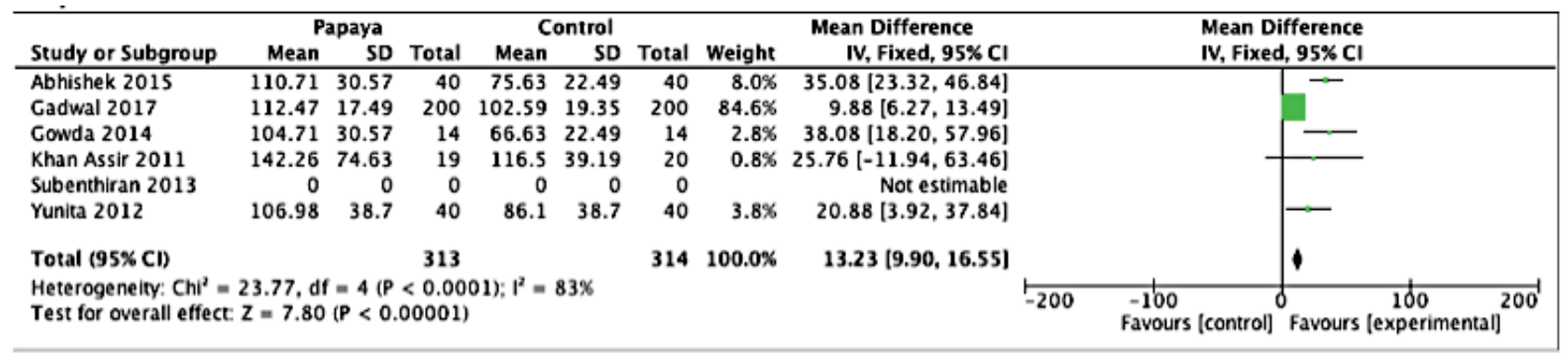

Figure 4. Efficacy of C. papaya leaf extract in increasing the platelet count measured at Day 5.

(MD: $13.23 ; \mathrm{Cl}=9.90,16.55)$ as compared to the control group. High heterogeneity was detected $\left(R^{2}=83 \%\right)$. The heterogeneity may be attributed to the differences among the study population, which include the factor that some of the participants may be on the resolution phase of the disease already at day 5 of treatment.

\section{Random Effects Model}

Due to the high heterogeneity detected (>75\%), random effects model was generated. The results still showed significant effect (MD=24.91; $C l=10.06$, 39.76) favoring the C. papaya group as compared to the control group

\section{Subgroup Analysis}

Subgroup analysis of four studies that used solid preparations of C. papaya leaf extract showed significant increase in platelet count in the experimental group ( $M D=24.89 ; \mathrm{Cl}: 8.73$, 41.06) compared to the control group. There was significant heterogeneity detected $\left(I^{2}=87 \%\right)$.

Subgroup analysis of four studies that administered the C. papaya treatment three times a day was also done. The results showed significant effect favoring the experimental group $(M D=31.61 ; C l=23.14$, 40.08) as compared to the control group. No heterogeneity was detected $\left(I^{2}=0 \%\right)$

Two of the included studies administered the C. papaya extract with the same frequency and dosage (1100 mg TID). Results showed significant effect favoring the experimental group in increasing the platelet count ( $M D=35.86, \mathrm{Cl}=25.74,45.98$ ) as compared to the control group. No heterogeneity was detected $\left(I^{2}=0 \%\right)$

\section{DISCUSSION}

After taking into account six randomized control trials, the review found that those who received papaya leaf extracts had higher platelet count than those who did not receive the extracts. The increase in platelet count was significant on the third, fourth and fifth day of administration. Pooled estimates on the fifth day showed beneficial effects of $C$. papaya extract using the random effects.

Heterogeneity may be due to differences between the studies included. Subgroup analysis was done based on the type of preparation (solid preparations) of C. papaya extract on the basis that the form in which the extract was given would affect absorption into the systemic circulation. Subgroup analysis still showed significant heterogeneity, probably due to the presence of inactive ingredients used in the formulation of the solid preparations of C. papaya extracts. Nevertheless, supplementation with the solid form preparation of C. papaya extract showed beneficial effect in increasing platelet count.

Another subgroup analysis was done pulling together studies that administered C. papaya extract in a three-times-a-day frequency since a higher dosing frequency would prevent fluctuations in the concentration of the extract, resulting to a longer duration of effect. With no heterogeneity found, the subanalysis showed beneficial effect in administering the C. papaya extract.

The last subgroup analysis pooled together studies which gave the same dose $(1100 \mathrm{mg})$ of C. papaya extract. Increasing the dose of a drug would increase the concentration of active ingredients in the body, which when above the minimum effective concentration, would increase the intensity of effect. The increase in dose may also lead to increased adverse effects and toxicity if any, depending on the properties of the extract. There was no heterogeneity in the analysis. The analysis showed beneficial effect in administering the C. papaya extract in doses of $1100 \mathrm{mg}$. Mild side effects such as nausea and vomiting were reported by the two studies who administered the extract in this dose and frequency. 
Results of the meta-analysis have shown a beneficial effect of $C$. papaya in increasing platelet count. This is in agreement with a recent meta-analysis done by Charan et. al in 2016, which involved only 4 trials present at the time, as it showed a definitive role of $C$. papaya leaf extracts in the improvement of platelet count in patients of dengue.

Dengue fever is an arthropod-borne viral infection caused by the dengue virus (DENV) with four related btut antigenically distinct serotypes catalyzed by viral-encoded RNA-dependent RNA polymerases (RdRps) and other cellular factors. These factors in turn cause an immune complex-mediated destruction of platelets and endothelial cells [10]. Indeed, antibodies to NS1 antigen have shown to cross react with these cells.

The active ingredients in C. papaya have been postulated to stabilize platelet membranes as well, hence, prevent platelet destruction. They contain flavonoids and has antioxidant and free radical scavenging activity, thereby stabilizing erythrocyte membranes and preventing lysis when subjected to stress such as infection [11]. According to studies, extract administration was also associated with elevation of white blood cell count and stabilization of hematocrit. Another plausible explanation for this would be the proven genetic ability of some ingredients in C. papaya to stabilize platelet membranes, notably, the upregulation of arachidonate 12-lipooxygenase (ALOX 12), which is known to increase megakaryocyte production, and platelet activating factor receptor (PTAFR) gene that expresses megakaryocytes, ultimately increasing the production of the precursor cells and their conversion into platelets [11].

\section{Limitations}

Potential limitation of this meta-analysis includes the different dose concentrations used in the studies. Another potential limitation is the baseline platelet count on which course of the disease process was obtained since different phases of the disease independently affect platelet count fluctuations.

\section{CONCLUSION}

Papaya extract may be considered a useful adjunct in the treatment of patients with dengue and dengue hemorrhagic fever, considering its wide availability locally, enhancing management for patients with possible bleeding episodes resulting in decreased hospital stay, complications, and morbidity and mortality rates. Being an addition to the limited pool of studies on the Carica papaya leaf extract, this may also ignite interest among other researchers to explore its beneficial effects, not only in the treatment of dengue, but also in treating other bleeding or platelet disorders. While we do not aim to replace the recommended guidelines on dengue treatment, the papaya extract may be used as an adjunct to avert the potentially lifethreatening consequences of thrombocytopenia.

\section{ACKNOWLEDGEMENTS}

No further acknowledgements.

\section{PERMISSION}

No special permissions needed.

\section{CONFLICT Of INTEREST STATEMENT}

The authors declare that there is no conflict of interest. 


\section{re) REFERENCES Cen}

[1] Singhi, S., Kissoon, N., \& Bansal, A. Dengue and dengue hemorrhagic fever: Management issues in an intensive care unit, J. Pediatrics. 2007; 83(2): 22-35.

[2] Guzman, M. G., Halstead, S. B., Artsob H., et al. Dengue: a continuing global threat, Nat Rev Microbiol. 2010; 8: 7-16.

[3] Department of Health, Epidemiology Bureau, Public Health Surveillance Division.

[4] Frances, E. E., Yara, A. H., Francisco, M. L., et al. Economic cost and burden of dengue in the Philippines, Am J Trop Med Hyg. 2015; 92(2): 360-366.

[5] Idrees, S., \& Ashfaq, U. A. A brief review on dengue molecular virology, diagnosis, treatment and prevalence in Pakistan, Genet Vaccines Ther. 2012; 10: 6.

[6] Yboa, B. C., \& Labrague, L. J. Dengue knowledge and preventive practices among rural residents in Samar Province, Philippines. Am J Public Health. 2013; 1(2): 47-52.
[7] Okeniyi, J. A., Ogunlesi, T. A., Oyelami, O. A., et al. Effectiveness of dried Carica papaya seeds against human intestinal parasitosis: a pilot study. J Med Food. 2007; 10: 194-196.

[8] Karishna, K. L., Paridhavi, M., \& Patel, J. A. Review on nutritional, medicinal and pharmacological properties of papaya. Nat Prod Radiance. 2008; 7: 364-373.

[9] Ranasinghe, P. R., Ranasinghe, P. A., Kaushalya, W. P., et al. In vitro erythrocyte membrane stabilization properties of Carica papaya leaf extracts. Phcog Res 2012; 4: 196-202.

[10] Sarala, N. \& Paknikar, S. S. Papaya extract to treat dengue: a novel therapeutic option? Ann Med Health Sci Res. 2014; 4(3): 320-324.

[11] Charan, J., Saxena, D., Goyal, J. P., et al. Efficacy and safety of Carica papaya leaf extract in the dengue: A systematic review and meta-analysis. Int J Appl Basic Med Res. 2016; 6(4): 249-254. 